\title{
Cognitive impairment among older adults living with HIV/AIDS and frailty
}

\author{
Alfonso Zamudio-Rodríguez ${ }^{1,2}$, Sara Aguilar-Navarro² and José Alberto Avila-Funes2,3 \\ ${ }^{1}$ Medical, Dental and Health Sciences Master's Degree and Doctorate Program, Clinical Epidemiology, Universidad Nacional Autónoma de México; \\ ${ }^{2}$ Department of Geriatrics, Instituto Nacional de Ciencias Médicas y Nutrición Salvador Zubirán, Mexico City, Mexico; ${ }^{3}$ Centre de Recherche Inserm, \\ U 1219, Bordeaux, F-33076, France
}

\begin{abstract}
In 2014, 17\% of newly diagnosed HIV infection cases in the United States were made in people over 50 years of age; actually, it is expected that in the near future this population group will be the most affected. This epidemiological change can be explained by the increased incidence of HIV infection in people over 50 years, but also by its higher prevalence due to treatment advances. As HIV infection has become a chronic one, new challenges have emerged. For instance, early-onset "geriatric syndromes," such as frailty, have been recognized in these patients. Frailty refers to a physiological state of vulnerability that increases the risk of adverse health-related outcomes. Frail individuals have higher risk of cognitive impairment; however, it is not known if early-onset frailty in those infected by HIV could also increase the risk of cognitive impairment in this already vulnerable population. The purpose of this review article is to describe, from an epidemiological point of view, the relationship between the changes promoted by HIV and the syndrome of frailty on cognitive function.
\end{abstract}

KEY WORDS: Frailty. HIV. AIDS. Cognitive impairment. Aging.

\section{Introduction}

Human immunodeficiency virus (HIV) infection and AIDS epidemiology has changed according to population aging ${ }^{1}$. According to US Centers for Disease Control and Prevention data, $17 \%$ of new cases of HIV infection in that country in 2014 corresponded to 50 year old and older people. Recently, the New York Medical Center reported that one out of every five new HIV-infection diagnoses was made in individuals older than 50 years ${ }^{2}$. This phenomenon has caused for the proportion of older adults diagnosed with HIV/AIDS to increase, which is the result of two main reasons: 1) an increased incidence of infection in this population that is not perceived as being at risk for sexually-transmitted infections, and 2) a drastic change in natural evolution of the disease, which has changed from being a disease that led to death to be a chronic disease ${ }^{3}$, mainly owing to the use of highly active antiretroviral therapy (HAART). This way, for 2015, 50\% of the population with HIV/AIDS was estimated to be 50 years' old or older, and to account for $15 \%$ of new diagnoses of infection with this virus in the American Union 4 .

The majority of older adults that are HIV carriers do not suspect they are infected ${ }^{5}$, and this is one of the reasons why the diagnosis is usually made at more advanced stages in comparison with younger patients ${ }^{6}$, which favors the development of comorbidity, lower treatment adherence and early appearance of geriatric syndromes. In this sense, HIV/AIDS is an "accelerated aging" model, since affected people can develop different syndromes that are traditionally seen at old age, even up to 15 years earlier than in non-infected people 7 . These include "frailty", which has important repercussions on older adults' health.

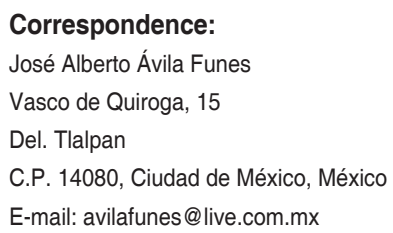

Date of reception: 14-09-2016

Date of acceptance: 15-05-2017

DOI://dx.doi.org/10.24875/GMM.M18000044
Gac Med Mex. 2017;153:544-552

Contents available at PubMed www.gacetamedicademexico.com 
The frailty syndrome describes a state of great vulnerability and poor resilience, the higher incidence of which is closely related to the passage of time, and its presence increases the risk of health adverse outcomes, such as disability, mortality and cognitive impairment ${ }^{8}$.

Just like frailty, cognitive function declination is an almost universal aging-related phenomenon'; however, HIV infection is also a condition that has been associated with the onset of cognitive changes. This way, a frailty, old age and HIV infection interaction is plausible, and it might favor a higher likelihood of cognitive impairment in people who suffer from them in comparison with those who don't have these problems. However, there is sparse information on the interaction of these three factors and the development of cognitive impairment. Therefore, the purpose of this review is to describe epidemiological evidence pointing at a possible relationship between HIV infection, frailty syndrome and cognitive changes in older adults.

\section{HIV infection and aging}

The human aging process results from an interrelation of genetic, biological, environmental and individual lifestyle factors ${ }^{10}$. HIV infection induces multiple changes in different organs and systems of the body, including the immune system, which is one of the most affected ${ }^{11-13}$. The virus promotes an inflammatory (C-reactive protein, interleukin 6, tumor necrosis factor alpha [TNF-a]) and procoagulant (fibrinogen, D-dimer) sustained response, which is related to viral load and is very similar to that described in non-VIH infected older people ${ }^{14,15}$.

On the other hand, there is also high toxicity generated by HAART chronic use, which was been associated with HIV patients' body vital capacity reduction, which may cause a multi-system damage that favors further deterioration ${ }^{16,17}$. However, HAART early initiation and managing to maintain CD4+ T-lymphocytes normal counts have been associated with better life expectan$c y$, very similar to that of the general population $n^{18,19}$.

The relationship between HIV and age has generated the concept of HIV-associated non-AIDS (HANA) conditions, which alternate with comorbidity in a common term. HANAs describe the multifactorial contribution of risk factors to the pathogenesis of concomitant diseases and, at the same time, they underscore that comorbidity clinical presentation is an intrinsic component of HIV-related disease in the HAART era. HANA conditions three pathogenic mainstays are: 1) HIV-related disease; 2) antiretroviral drugs toxicity; and 3) host-associated risk factors ${ }^{20}$. This biological process generates a higher number of chronic conditions that can occur simultaneously, such as cardiovascular disease, hypertension, renal impairment and diabetes, among others, which largely lower quality of life ${ }^{7}$. In addition, these conditions have shown higher prevalence at earlier stages of life in comparison with subjects without HIV of the same age ${ }^{21}$. Pluripathology is a risk factor for polypharmacy, which in turn increases the likelihood of toxicity and drug-drug interactions that may predispose to other conditions (arrhythmia, falls, depression, renal impairment, etc.) ${ }^{22}$.

On the other hand, HIV-infected population is known to have high prevalence of addictions and use of drugs, both licit and illicit. An example is smoking (three-fold more common), which entails an increased risk for suffering acute myocardial infarction, lung cancer, emphysema or stroke. In addition, the use of other substances by these individuals, such as amphetamines and cocaine, has been implied in problems of memory, bone mineral loss and heart conditions, which in combination favors adverse outcomes ${ }^{23}$. In this sense, the increase of older population with HIV represents a challenge for the different world health systems, as they are dealing with populations at high risk for developing a large number of medical conditions driven both by age and the virus itself. Therefore, creating initiatives and actions that adapt to this population's needs and that include a delay in the development of geriatric syndromes is necessary.

\section{Frailty in individuals with HIV}

Frailty is a state of great vulnerability that increases the risk for developing adverse health outcomes and that is different from disability and comorbidity ${ }^{8}$. Although the consequences for health can be disastrous, it is a potentially reversible and preventable process ${ }^{24}$. The frailty concept -considered as a syndrome- applied to the HIV-infected individual was initially studied in the Multicenter AIDS Cohort (MACS), where the prevalence and expression of frailty was studied in a cohort of 1946 men (mean age, 53.8 years; 898 HIV+ with HAART use and 1048 HIV-) between 2007 and 2011. The investigators reported a phenotype that was similar to that described in older adults (frailty-like) in 477 patients, out of which $54 \%$ were $\mathrm{HIV}^{25}$. Desquilbet et al. ${ }^{26}$, with a sample of 2150 participants (mean age, 42 years, range, 37 to 47 ), reported a prevalence of frailty of $13.9 \%{ }^{26}$. Terzian et al. ${ }^{27}$, in a sub-analysis of the Women's Interagency HIV Study (WIHS) (a prospective cohort study started in 1994 in five cities of 
the USA) with 1781 female participants (573 HIV- and $1206 \mathrm{HIV+}$ ), all younger than 50 years, demonstrated a prevalence of frailty of $8 \%$ in HIV- women in comparison with $12 \%$ in those who were HIV+, but it was even higher $(20 \%)$ in those with CD4 T-lymphocyte count $<100 \mathrm{cell} / \mathrm{mm}^{327}$. Onen et al. ${ }^{28} \mathrm{cross}-$ sectional analysis of the Study to Understand the Natural History if HIV and AIDS in the Era of Effective Therapy (SUN) (a cohort of 322 participants with HIV, mean age of 47 years and $79 \%$ men) determined a prevalence of frailty of $5 \%$ according to the Cardiovascular Health Study criteria.

The presence of frailty in the population with HIV has been inversely related to the use of HAART. Desquilbet et al. ${ }^{29}$ reported a drop in the prevalence of frailty of more than one half (from 24 to $10.1 \%$ ) after the introduction of HAART, which turned out to be one of the main protecting factors against the development of frailty ${ }^{30-32}$. However, there is a mismatch in the age of onset of this geriatric syndrome, since its prevalence in the 55-year old population with HIV appears to be similar to that reported in subjects older than 65 years without $\mathrm{HIV}$; this observation reinforces the idea of frailty early onset in this population, probably owing to conditions promoted by the virus ${ }^{30}$. This way, the frequency of frailty syndrome among relatively young HIV-infected individuals appears to be similar to that reported by epidemiological studies that use to include 65-year old and older adults without infection ${ }^{8,33}$. In the light of this evidence, HIV infection appears to be a frailty-promoting factor in this population, regardless of people's age.

\section{Frailty and cognitive impairment}

In spite of the difficulties to find a universal definition of frailty, there is a consensus that this state is the cause of multiple adverse outcomes, including a poor quality of life, greater disability, more hospitalizations, higher probability to be institutionalized and higher risk of dying ${ }^{8,34}$. The mechanisms that link cognitive impairment and frailty might be associated with endothelial dysfunction within a proinflammatory environment with increased oxidative stress ${ }^{35}$. On the other hand, aging-associated atherosclerotic processes, which can even produce cerebrovascular events, are interrelated with frailty by means of a series of factors such as inflammation, procoagulant processes and fibrinolytic systems ${ }^{37}$, which places frailty expression as a possible prodromal stage of vascular-cause cognitive impairment. Frailty is related to multiple chronic conditions and functional deterioration, which requires a larger amount of energy; thus, this condition might explain why mitochondrial metabolism produces higher quantities of free radicals. At the same time, this increase in the production of free radicals can also activate nuclear factor kappa $B$ pathway, which in turn leads to inflammation ${ }^{36}$. Frailty most popular phenotype is the one proposed by Fried et al. ${ }^{8}$, which has demonstrated its validity in the prediction of health-adverse outcomes; however, the most important criticism to this phenotype is that its five components are of physical nature, and other potential components that are also usually affected by aging have therefore been proposed. Accumulation of deficits over time ("frailty index") is one of them, which weighs the presence of multiple common problems at old age (such as functional status, comorbidity or psychosocial factors) and, the higher the problem accumulation, the higher the risk for adverse outcomes ${ }^{37}$. However, cognitive function changes (an almost universal phenomenon that occurs with aging) have been inconsistently included in frailty definitions. In recent years, understanding of the relationship between frailty and the development of cognitive impairment has improved.

Frail adults often have poorer performance in cognitive tests they undergo. For example, in a study on the association between frailty and cognitive impairment conducted in 155 older adults without dementia (mean age of $67.4 \pm 5.4$ years), those who were frail had poorer performance in different neurocognitive tests, such as the mini-mental state examination (MMSE) and the Montreal Cognitive Test (MoCA). In this study, frailty measured with the Edmonton Frail Scale had a negative and statistically significant correlation with the degree of cognitive performance, as shown with the used tests (MMSE: $r=-0.622, p<0.001$; MoCA: $r=-0.687, p<0.001$ ), which indicates that cognitive performance declines as frailty increases ${ }^{38}$.

Frailty has been associated with the development of different degrees of cognitive impairment. Boyle et al. ${ }^{39}$ reported the relationship between a frailty scale (measured in the grip strength, gait velocity, body mass index and extenuation components) and the development of mild cognitive impairment in 761 subjects (mean age, $79 \pm 7.1$ years). During the 12-year follow-up, $40 \%$ developed mild cognitive impairment, and the risk for it increased by up to $63 \%$ for each unit of increase in the frailty scale (hazard-ratio [HR]: 1.63; $95 \%$ confidence interval [Cl]: 1.27-2.08) ${ }^{39}$. Another cohort study with a 4-year follow-up carried out in a French population (6030 participants; mean age, 74.1 \pm 5.2 years; $61.2 \%$ females) showed that frailty was a risk factor for dementia. However, risk was statistically significant only in those people with poor cognitive 
performance (but not dementia) at study enrollment, regardless of their physical status (HR: $4.98 ; 95 \% \mathrm{Cl}$ : $2.17-11.41 ; p<0.001)^{8}$. On the same token, Samper-Ternent et al..$^{40}$ reported, with information of 1370 65-year old and older participants (mean age, $73.2 \pm 4.8$ years) from the Hispanic Established Population for the Epidemiological Study of the Elderly (H-EPESE), that those who were classified as frail at study inclusion were at higher risk for cognitive impairment in comparison with those who were not frail, after 10 years of follow-up (odds ratio [OR]: $1.27 ; 95 \% \mathrm{Cl}: 1.07-1.52)^{40}$. These observations point at frailty as a state that drives to cognitive impairment, although the pathophysiological pathways have not been clearly elucidated. Probably some inflammatory mechanisms are implicated in the relationship of both problems, as well as vascular changes, which can favor cognition impairment. This statement results from the conclusions of two epidemiological studies that point at frailty as a risk factor for vascular-type dementia ${ }^{41,42}$. One of them included 5248 participants (from 65 to 95 years) and focused on the study of the relationship between this geriatric syndrome and the incidence of vascular-type dementia. After 7 years of follow-up, those subjects who were frail at study inclusion and had no dementia, were at higher risk for developing vascular dementia (HR: 2.73; 95\% Cl: 1.05-7.13) even after adjusting for multiple confounders; this study concluded that frailty is a major risk factor for the development of this type of dementia ${ }^{41}$. The same finding was replicated by an Italian study with a mean follow-up of 3.5 years, where frailty was found to be a risk factor for vascular-type dementia as well, even after adjustment for potential confounders (HR: 2.68; 95\% Cl: 1.16-7.17) ${ }^{42}$. In the light of these results, frailty expression has been considered to be a prodromal stage of vascular dementia, which is supported by the results of a study that shows that vascular changes, such as carotid intima-media increased thickness, as well as carotid lumen increased diameter (as shown by Doppler ultrasound), are more common in frail than non-frail individuals ${ }^{43}$.

Frailty is a risk factor for cognitive impairment, especially of that of the vascular type. Early identification of this geriatric syndrome might be useful in an effort to modify its possible evolution towards individual cognitive status decline.

\section{Cognitive impairment in people with HIV}

The introduction of HAART has radically modified vital and functional prognosis of HIV-infected people.
In this sense, one benefit observed in the HIV+ population has been an improvement in their cognitive status, since in the pre-HAART era usually they progressed towards impairment. However, this good result has not been consistent, since $50 \%$ of HIV+ patients still have neurocognitive disorder associated with the virus (known as HAND) ${ }^{44}$.

The central nervous system (CNS) is particularly affected by HIV, and the damage has been demonstrated both at its structure and function. One study that reported brain histopathology findings of 390 HIV-infected subjects, carried out at the San Diego Medical Center of the California University, showed great alterations of this organ, with HIV-associated encephalitis and opportunistic infections being the conditions that most affected the $\mathrm{CNS}^{45}$. There is no particular area of the encephalon that is specifically harmed by HIV; however, some areas can be more affected than others, such as the temporal white matter (especially the hippocampus) and the parietal cortex ${ }^{46}$. The HIV Neurobehavioral Research Center reported the impact of HIV infection-associated cognitive impairment on daily life functions in a sample of 267 $\mathrm{HIV}+$ participants (mean age of $39.3 \pm 7.5$ years and mean education level of 13.6 years). The cognitive domains that were most compromised in HIV-infected subjects were shown to be those associated with learning, executive functions/abstraction, attention/ working memory and motor functions (Table 1). On the other hand, less compromised areas were those associated with information-processing speed, verbal functions and deferred memory ${ }^{47}$. Studies conducted with magnetic resonance have shown flow alterations in microstructures around the white matter in brains of HIV-infected individuals (e.g. mean dispersion increase and anisotropic fraction reduction) $)^{48}$. Specifically, the presence of these changes in brain microstructures has been associated with psychiatric (major depressive disorder, anxiety, etc.) and neurologic conditions (neurocognitive disorders, delirium or HAND) in HIV-infected persons ${ }^{49,50}$.

HIV is a neurotropic virus that lodges in the immune system, promoting the production of neurotoxic substances such as quinolinic acid and some excitatory amino acids (L-cysteine, glutamate, arachidonic acid, free radicals and TNF- $\alpha$, among others). These factors, originating from macrophages and possibly astrocytes, contribute to neuronal damage, especially to the damage of dendrite synapses and inducing their apoptosis. HIV introduction into monocytes via gp120 triggers a series of events that start with the 

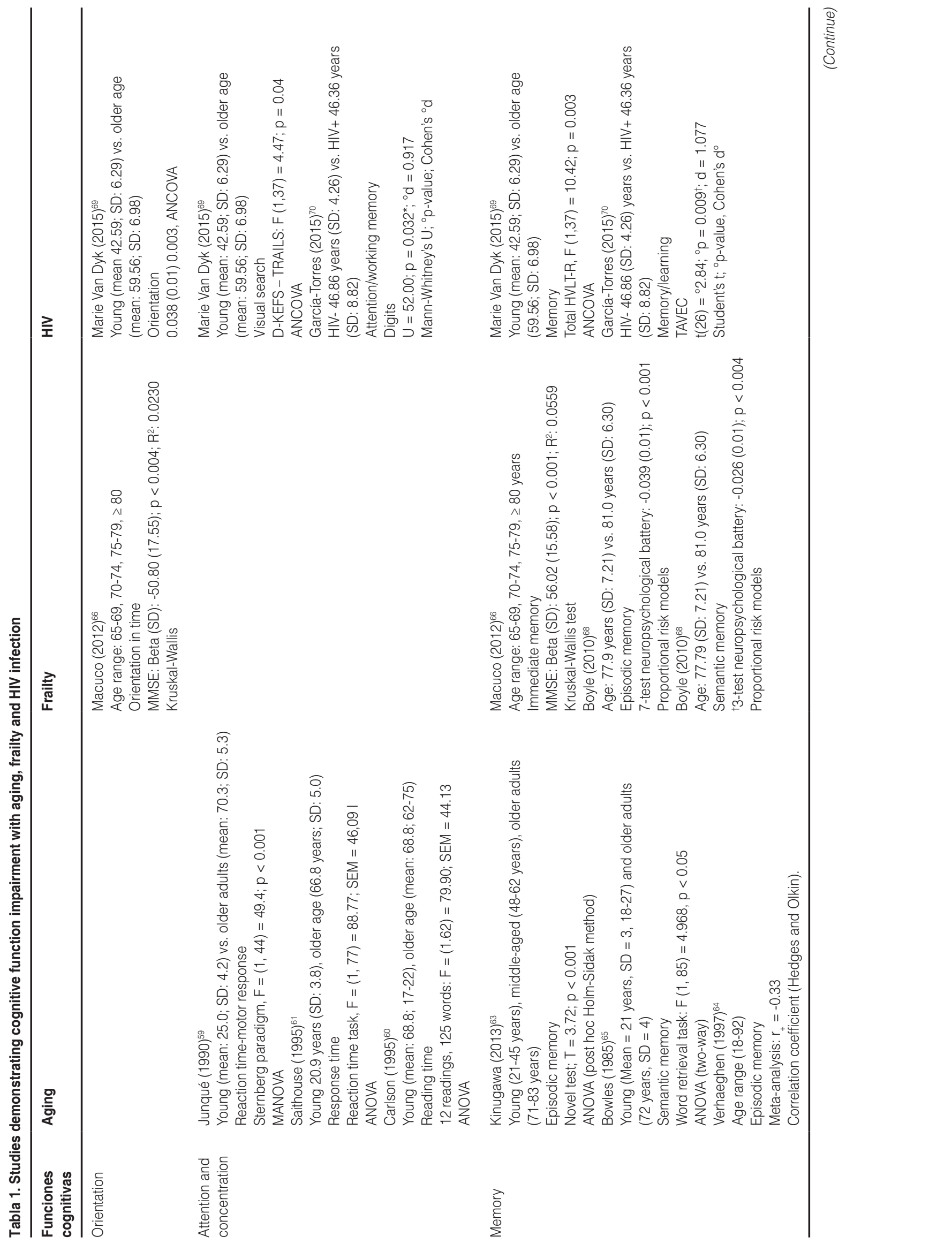


Tabla 2. HIV-associated neurocognitive disorder (modified from Antinori A et al. ${ }^{58}$ )

\begin{tabular}{ll}
\hline Diagnosis & Criteria \\
\hline $\begin{array}{l}\text { Asymptomatic neurocognitive } \\
\text { impairment }\end{array}$ & $\begin{array}{l}\text { Impairment on at least two cognitive domains assessed by means of a neuropsychological test } \\
\text { standardized by gender, age and level of education (at least one standard deviation and without } \\
\text { functional deterioration) }\end{array}$ \\
Mild neurocognitive impairment & $\begin{array}{l}\text { Impairment on at least two cognitive domains assessed by means of a neuropsychological } \\
\text { test standardized by gender, age and level of education (at least one standard deviation; slight } \\
\text { interference with daily life activities) }\end{array}$ \\
HIV-associated dementia & $\begin{array}{l}\text { Severe impairment on at least two cognitive domains assessed by means of a neurocognitive } \\
\text { test standardized by gender, age and level of education (at least two standard deviations), with } \\
\text { marked impact on daily functions }\end{array}$ \\
\hline
\end{tabular}

production of TNF- $\alpha$ and interleukin $1 \mathrm{~b}$, which in turn activate astrocytes, which release glutamate and nitric oxide radicals, which in turn react with superoxide and generate neurotoxic molecules; in addition, nitric oxide can activate extracellular matrix metalloproteases that favor neuronal damage and ultimately proteolysis, which causes direct damage at the CNS ${ }^{51}$.

On the other hand, a relationship has been demonstrated between the degree of disease progression and cognitive impairment severity. Therefore, the prevalence of cognitive impairment, for example, at stage C, was higher in the pre-HAART era $(52 \%)$ and lower after this therapy became available $(45 \%)^{52}$.

In 1991, the American Academy of Neurology AIDS Task Force proposed the nomenclature and diagnostic criteria for HIV-1-infection neurological manifestations. Years later, after HAART implementation, they were adapted and the inclusion of the term "asymptomatic neurocognitive impairment" was suggested to identify those individuals not displaying overt cognitive impairment, but who showed abnormal performance in standard neuropsychological tests. In order to enable establishing the diagnosis of HIV-associated neurocognitive disorders, changes to these criteria were proposed, also introducing functional performance assessment as a parameter of HIV-related neurocognitive disorder progression (Table 2). In this sense, it is recommended that HIV patient neurocognitive evaluation should take the following functions into account: attention/working memory, information-processing speed, verbal memory, learning, verbal fluency, executive functions and motor function, the results of which should consider subjects' age and level of education and agree with existing regulatory data $^{53}$. HIV-associated neurocognitive disorders are often the consequence of viral replication in the CNS, which per se is already compromised by an inflammatory response, but that in the past few years has been attenuated thanks to HAART.
The presence of neurocognitive problems in HIV patients has other clinical implications. Those subjects with some degree of impairment might have lower treatment adherence and more disability for their daily activities, in addition to the loss of their working activities, deterioration of quality of life in general and increased risk of death. Since HIV-related cognitive damage is a risk factor for adverse outcomes on infected individuals' health and quality of life, systematic cognitive evaluation of these patients should be considered in order to enable the implementation of opportune intervention measures according to their needs.

\section{Cognitive impairment in individuals with HIV and frailty}

Several studies have suggested that suffering from any form of HAND is associated with the frailty phenotype in HIV patients, as demonstrated in the MACS study. In a retrospective sub-analysis of this cohort, which included 505 participants (mean age 52 years, range from 32 to 69 , and $36.9 \%$ with $\geq 16$ years of education), $12.7 \%$ had asymptomatic cognitive impairment, $36.6 \%$ had symptomatic cognitive impairment, $12.9 \%$ had HIV-associated dementia and the rest had no impairment. It was established that those with some type of HAND had twice the risk to develop frailty (three of the five components proposed by Fried et al. ${ }^{8}$ ) (OR: 2.18; 95\% Cl: 1.05-4.54; $p=0.036$ ), whereas in individuals who showed any HAND symptomatic form (either symptomatic cognitive impairment or HIV-associated dementia) the risk for developing frailty was up to 3-fold higher (OR: 2.99; 95\% Cl: 1.49-5.96; $p=0.002$ ). In a second analysis adjusted by age, ethnicity, level of education, employment, CD4 load, smoking, use of marihuana, use of intravenous drugs and depression, the risk for the development of frailty was maintained among those who had HAND (OR: 2.2; 95\% Cl: 1.034.68; $p=0.042$ ) or symptomatic HAND (OR: $2.8 ; 95 \%$ 
Cl: $1.37-5.75 ; p=0.005)$. These results support the idea of a significant relationship existing between frailty and the presence of HAND; however, these findings have to be replicated in similar populations. With these results, it would appear necessary recommending systematic serial cognitive evaluations in individuals that are frail and that are infected with HIV ${ }^{54}$.

The presence of cognitive impairment can be associated with functional impairment in HIV-infected individuals. This was shown in the Geriatric HIV Program: The Experience of an Urban Academic Center at One Year Follow-Up study, carried out at the Medical Center of Louisiana, in New Orleans. A total of 60 patients were evaluated in order to determine the presence of frailty-like syndrome (which considered the cognition, daily life basic and instrumented activities, nutritional status, depression, hearing loss, visual deficit and mobility); of them, only 20 entered the program (12 men and 8 women with a mean age of 63.5 years) and were divided into three subgroups according to their degree of frailty: mild $(20 \%)$ when only one domain was compromised, moderate $(50 \%)$ with two affected domains and severe $(30 \%)$ with three or more involved domains. In this study, at one year follow-up, half the participants developed some type of cognitive impairments, and those with higher degree of frailty were the most affected, which supports the idea of a relationship between frailty intensity and the degree of cognitive impairment in individuals with $\mathrm{HIV}^{55}$. In the same sense, the study by the Hospital HIV Clinic and the San Francisco Veterans Affairs Medical Center Infectious Diseases Clinic (155 participants, $93.6 \%$ males, with a mean age of 57 years) showed the presence of frailty in $9 \%$ and cognitive impairment in $46.7 \%$, which is a reflection of the high prevalence of both these conditions commonly seen in the elderly, but also among a relatively young HIV+ population ${ }^{56}$. Previous studies support the hypothesis that frailty syndrome can be observed in the HIV+ population regardless of age. This way, HIV+ patients are more prone to develop more health-adverse outcomes, including higher institutionalization, more hospital admissions and even higher probability of death. Since frailty is a state of risk for the development of cognitive impairment, it is plausible that the presence of frailty syndrome can promote a more accentuated or accelerated cognitive impairment in HIV-infected persons, in comparison with those fragile individuals without HIV infection. Frailty early identification in people with HIV can be useful to deliberatively investigate the presence of any type of cognitive impairment. This opens the possibility for the implementation of early interventions with the purpose to modify the course of frailty and avoid its disastrous complications. Owing to the paucity of scientific information, promoting studies better looking into the possible interaction of frailty and HIV infection with cognitive impairment, as well as other geriatric syndromes, early onset in younger populations is desirable.

\section{Conclusions}

HIV infection epidemiological change entails new challenges for health systems. Anatomical and physiological changes promoted by aging, together with the effects of HIV on the immune system, generate a state of increased vulnerability that has been shown to be related to the onset of frailty and its adverse outcomes, including cognitive impairment. However, the pathophysiological pathways implicated in these complications still remain to be determined. Both conditions' inflammatory mechanisms open the possibility for vascular alterations to be the cause of the compromise of cognitive function in frail subjects, just as observed in those without HIV. However, peculiarities of this chronic infection, as well as the presence of factors such as HAART or comorbidity in an increasingly older population, might play a fundamental role in cognitive impairment early or magnified onset in infected people. Treatment and health-associated preventive measures that are applied in older adults with frailty require to be extended to aging patients with HIV, since their efficacy and impact on this already vulnerable population is not known. Doing it might reduce the presence of HIV-associated cognitive alterations.

\section{References}

1. Nguyen N, Holodniy M. HIV infection in the elderly. Clin Interv Aging. 2008;3:453-72.

2. Bernstein L. The graying of HIV: 1 in 6 new U.S. cases are people older than 50. The Washington Post. 2016. Disponible en: www.washingtonpost.com/national/health-science/the-graying-of-hiv-1-in-6-new-us-cases-are-people-older-than-50/2016/04/05/089cd9aa-f68a-11e5-8b23538270a1ca31_story.html.

3. Deeks SG, Lewin SR, Havlir DV. The end of AIDS: HIV infection as a chronic disease. Lancet. 2013;382:1525-33.

4. Centers for Disease Control and Prevention - CDC (USA). Persons aged 50 and older. Disponible en: http://www.cdc.gov/hiv/topics/over50/index.htm

5. Abel T, Werner M. HIV risk behaviour of older persons. Eur J Public Health. 2003;13:350-2.

6. Centers for Disease Control and Prevention (CDC). AIDS among persons aged $>$ or $=50$ years - United States, 1991-1996. MMWR Morb Mortal Wkly Rep. 1998;47:21-7.

7. Guaraldi G, Orlando G, Zona S, et al. Premature age-related comorbidities among HIV-infected persons compared with the general population. Clin Infect Dis. 2011;53:1120-6.

8. Fried LP, Tangen CM, Walston J, et al. Frailty in older adults: evidence for a phenotype. J Gerontol A Biol Sci Med Sci. 2001;56:M146-56.

9. Bergman H, Ferrucci L, Guralnik J, et al. Frailty: an emerging research and clinical paradigm - issues and controversies. J Gerontol A Biol Sci Med Sci. 2007;62:731-7. 
10. Manton KG. Dynamic paradigms for human mortality and aging. J Gerontol A Biol Sci Med Sci. 1999;54:B247-54.

11. Hazleton JE, Berman JW, Eugenin EA. Novel mechanisms of CNS damage in HIV infection. HIV AIDS (Auckl). 2010;2:39-49.

12. Clifford DB. HIV-associated neurocognitive disease continues in the antiretroviral era. Top HIV Med. 2008;2:94-8.

13. Padilla L, Martin HO, Iguain JL. Anomalous diffusion with log-periodic modulation in a selected time interval. Phys Rev E Stat Nonlin Soft Matter Phys. 2011;83(2 Pt 1):020105.

14. Shlipak MG, Fried LF, Crump C, et al. Elevations of inflammatory and procoagulant biomarkers in elderly persons with renal insufficiency. Circulation. 2003;107:87-92.

15. Deeks SG. HIV infection, inflammation, immunosenescence, and aging Ann Rev Med. 2011;62:141-55.

16. Fellay J, Boubaker K, Ledergerber B, et al. Prevalence of adverse events associated with potent antiretroviral treatment: Swiss HIV Cohort Study. Lancet. 2001;358:1322-7.

17. Reisler RB, Han C, Burman WJ, et al. Grade 4 events are as important as AIDS events in the era of HAART. J Acquir Immune Defic Syndr. 2003;34:379-86.

18. May MT, Ingle SM. Life expectancy of HIV-positive adults: a review. Sex Health. 2011:8:526-33.

19. Bendavid E, Ford N, Mills EJ. HIV and Africa's elderly: the problems and possibilities. AIDS. 2012;26(Suppl 1):S85-91.

20. Hasse B, Bernasconi E, Furrer $\mathrm{H}$, et al. [HIV-associated non-AIDS conditions]. Therapeutische Umschau Revue Therapeutique. 2014;71:483-9.

21. Deeks SG, Phillips AN. HIV infection, antiretroviral treatment, ageing, and non-AIDS related morbidity. BMJ. 2009;338:a3172.

22. Gleason LJ, Luque AE, Shah K. Polypharmacy in the HIV-infected older adult population. Clin Interv Aging. 2013;8:749-63.

23. HIV in specific populations. HIV and aging. POZ. 2016, February 14. Disponible en: https://www.poz.com/basics/hiv-basics/hiv-aging

24. Gill TM, Gahbauer EA, Allore HG. Transitions between frailty states among community-living older persons. Arch Intern Med. 2006;166:418-23.

25. Althoff KN, Jacobson LP, Cranston RD, et al. Age, comorbidities, and AIDS predict a frailty phenotype in men who have sex with men. J Gerontol A Biol Sci Med Sci. 2014;69:189-98.

26. Desquilbet L, Jacobson LP, Fried LP, et al. HIV-1 infection is associated with an earlier occurrence of a phenotype related to frailty. J Gerontol A Biol Sci Med Sci. 2007;62:1279-86.

27. Terzian AS, Holman S, Nathwani N, et al. Women's Interagency HIV Study. Factors associated with preclinical disability and frailty among HIV-infected and HIV uninfected women in the era of cART. J Womens Health 2009:18:1965-74.

28. Onen NF, Patel P, Baker J, et al. Frailty and pre-frailty in a contemporary cohort of HIV-infected adults. J Frailty Aging. 2014;3:158-65.

29. Desquibet L, Margolick JB, Fried LP, et al. Relationship between a frailty-related phenotype and progressive deterioration of the immune system in HIV-infected men. J Acquir Immune Defic Syndr. 2009;50:299-306.

30. Rees $\mathrm{HC}$, lanas $\mathrm{V}, \mathrm{McC}$ racken $\mathrm{P}$, et al. Measuring frailty in HIV-infected individuals. Identification of frail patients is the first step to amelioration and reversal of frailty. J Vis Exp. 2013;(77).

31. Lee DH, Buth KJ, Martin BJ, et al. Frail patients are at increased risk for mortality and prolonged institutional care after cardiac surgery. Circulation. 2010;121:973-8.

32. Woods NF, LaCroix AZ, Gray SL, et al. Frailty: emergence and consequences in women aged 65 and older in the Women's Health Initiative Observational Study. J Am Geriatr Soc. 2005;53:1321-30.

33. Avila-Funes JA, Helmer $\mathrm{C}$, Amieva $\mathrm{H}$, et al. Frailty among community-dwelling elderly people in France: the three-city study. J Gerontol A Biol Sci Med Sci. 2008:63:1089-96.

34. Avila-Funes JA, Amieva H, Barberger-Gateau P, et al. Cognitive impairment improves the predictive validity of the phenotype of frailty for adverse health outcomes: the three-city study. J Am Geriatr Soc. 2009; 57:453-61

35. Rodriguez-Manas L, El-Assar M, Vallejo S, et al. Endothelial dysfunction in aged humans is related with oxidative stress and vascular inflammation. Aging cell. 2009;8:226-38.

36. Piette J, Piret B, Bonizzi G, et al. Multiple redox regulation in NF-kappaB transcription factor activation. Biol Chem. 1997;378:1237-45.

37. Mitnitski AB, Mogilner AJ, Rockwood K. Accumulation of deficits as a proxy measure of aging. ScientificWorldJournal. 2001;1:323-36

38. Otassem S. Amer, Rania M. El Akkad, Heba S. Hassan Association of cognitive impairment with frailty in elderly: a cross-sectional study on communitydwelling non demented elderly referred to an outpatient geriatric service in Egypt. Indian Journal of Applied Research, Vol.5, Issue: 9 September 2015.

39. Boyle PA, Buchman AS, Wilson RS, et al. Physical frailty is associated with incident mild cognitive impairment in community-based older persons. J Am Geriatr Soc. 2010;58:248-55.
40. Samper-Ternent R, Al Snih S, Raji MA, et al. Relationship between frailty and cognitive decline in older Mexican Americans. J Am Geriatr Soc. 2008;56:1845-52.

41. Avila-Funes JA, Carcaillon L, Helmer C, et al. Is frailty a prodromal stage of vasculardementia? Results from the three-city study. J Am Geriatr Soc. 2012;60:1708-12.

42. Solfrizzi V, Scafato E, Frisardi V, et al. Frailty syndrome and the risk of vasculardementia. The Italian Longitudinal Study on Aging. Alzheimers Dement. 2013:9:113-22.

43. Avila-Funes JA, Meillon C, Gonzalez-Colaco Harmand M, et al. Association between frailty and carotid central structure changes: the Three-City Study. J Am Geriatr Soc. 2014:62:1906-11.

44. Cody SL, Vance DE. The neurobiology of HIV and its impact on cognitive reserve: a review of cognitive interventions for an aging population. Neurobiol Dis. 2016:92(Pt B):144-56.

45. Masliah E, DeTeresa RM, Mallory ME, et al. Changes in pathological findings at autopsy in AIDS cases for the last 15 years. AIDS. 2000;14:69-74.

46. Thompson PM, Dutton RA, Hayashi KM, et al. Thinning of the cerebral cortex visualized in HIV/AIDS reflects CD4+ T lymphocyte decline. Proc Nat Acad Sci U S A. 2005;102:15647-52.

47. Heaton RK, Marcotte TD, Mindt MR, et al. The impact of HIV-associated neuropsychological impairment on everyday functioning. J Int Neuropsychol Soc. 2004;10:317-31.

48. Pfefferbaum A, Rosenbloom MJ, Adalsteinsson E, et al. Diffusion tensor imaging with quantitative fibre tracking in HIV infection and alcoholism comorbidity: synergistic white matter damage. Brain. 2007;130(Pt 1):48-64.

49. Watkins CC, Treisman GJ. Neuropsychiatric complications of aging with HIV. J Neurovirol. 2012;18:277-90.

50. Wendelken LA, Valcour V. Impact of HIV and aging on neuropsychological function. J Neurovirol. 2012;18:256-63.

51. Kaul M, Zheng J, Okamoto S, et al. HIV-1 infection and AIDS: consequences for the central nervous system. Cell Death and Differentiation. 2005;12(Suppl 1):878-92.

52. Heaton RK, Clifford DB, Franklin DR, Jr., et al. HIV-associated neurocognitive disorders persist in the era of potent antiretroviral therapy: CHARTER Study. Neurology. 2010;75:2087-96.

53. Schouten J, Cinque P, Gisslen M, et al. HIV-1 infection and cognitive impairment in the CART era: a review. AIDS. 2011;25:561-75.

54. Smith B Skolasky R SO, et al. Association of HIV-associated neurocognitive disorder with frailty in HIV-1 seropositive men. 3rd International Workshop on HIV and Aging; 2012:5-6.

55. Ruiz M, Cefalu C. Characteristics of frail patients in a geriatric-HIV program: the experience of an urban academic center at one year follow-up. $\mathrm{J}$ Int Assoc Physicians AIDS Care (Chic). 2011;10:138-43.

56. Greene M, Valcour V. Geriatric syndromes in older HIV-infected adults. J Acquir Immune Defic Syndr. 2015:69:161-7.

57. Antinori A, Arendt G, Becker JT, et al. Updated research nosology for HIV-associated neurocognitive disorders. Neurology. 2007;69:1789-99.

58. Junqué $C$, Jódar M. Velocidad de procesamiento cognitivo en el envejecimiento. Anales de Psicología 1990;6:199-207.

59. Carlson MC, Hasher L, Zacks RT, et al. Aging, distraction, and the benefits of predictable location. Psychol Aging. 1995;10:427-36.

60. Salthouse TA, Fristoe NM, Lineweaver TT, et al. Aging of attention: does the ability to divide decline? Memory and Cognition. 1995;23:59-71.

61. Salthouse TA, Mitchell DRD, Skovronek E, et al. Effects of adult age and working memory on reasoning and spatial abilities. J Exp Psychol Learn Mem Cogn. 1989:15:507-16.

62. Kinugawa K, Schumm S, Pollina M, et al. Aging-related episodic memory decline: are emotions the key?; Front Behav Neurosci. 2013;7:2.

63. Verhaeghen P, Salthouse TA. Meta-analyses of age-cognition relations in adulthood: estimates of linear and nonlinear age effects and structural models. Psychol Bull. 1997;122:231-49.

64. Bowles NL, Poon LW. Aging and retrieval of words in semantic memory. J Gerontol. 1985:40:71-7.

65. Macuco CR, Batistoni SS, Lopes A, et al. Mini-Mental State Examination performance in frail, pre-frail, and non-frail community dwelling older adults in Ermelino Matarazzo, São Paulo, Brazil. Int Psychogeriatr. 2012;24:1725-31.

66. Langlois F, Vu TT, Kergoat MJ, et al. The multiple dimensions of frailty: physical capacity, cognition, and quality of life. Int Psychogeriatr. 2012;24:1429-36.

67. Boyle PA, Buchman AS, Wilson RS, et al. Physical frailty is associated with incident mild cognitive impairment in community-based older persons. J Am Geriatr Soc. 2010;58:248-55.

68. Van Dyk KM. Attention in HIV (2015). CUNY Academic Works. Disponible en: http://academicworks.cuny.edu/gc_etds/633

69. García-Torres E, Vergara-Moragues A, Pinón-Blanco M, et al. Alteraciones neuropsicológicas en pacientes con $\mathrm{VIH}$ e historia previa de consumo de sustancias. Un estudio preliminar. Revista Latinoamericana de Psicología. 2015;47:213-21. 